\title{
Differences in central facilitation between episodic and chronic migraineurs in nociceptive-specific trigeminal pathways
}

\author{
Jong-Hee Sohn ${ }^{*}$, Chul-Ho Kim and Hui-Chul Choi
}

\begin{abstract}
Background: The trigeminal nociceptive system plays a pivotal role in the pathophysiology of migraines. The present study investigated whether there are differences between patients with episodic migraine (EM) and patients with chronic migraine $(C M)$ in trigeminal pain processing at the brainstem and cortical levels using the nociceptive blink reflex (nBR) and pain-related evoked potentials (PREP).

Methods: This study assessed 68 female migraineurs (38 EM patients and 30 CM patients) and 40 age-matched controls using simultaneous recordings of $\mathrm{nBR}$ and PREP during the interictal period.

Results: In terms of the nBR, EM patients displayed significantly decreased latencies and larger amplitudes and area-under-the-curve (AUC) values for the R2 component, whereas CM patients showed significantly prolonged latencies and smaller amplitudes and AUC values for the R2 component $(p<0.05)$. In terms of PREP, both the EM and CM patients had decreased latencies (N1, P1), with larger amplitude compared with the controls $(p<0.05)$, which indicates facilitation at the cortical level. Additionally, the amplitude and AUC values of the R2 component exhibited a negative correlation, whereas the latency of the R2 component for the nBR showed a positive correlation, with the frequency of headaches in migraineurs $(p<0.01)$.

Conclusions: In the present study, the facilitation in the trigeminal nociceptive pathway of the EM group suggests the occurrence of migraine-specific hyperexcitability. Additionally, the suppression of R2 at the brainstem level in the CM group may relate to impaired or dysfunctional descending pain modulation. These findings suggest that there are adaptive or maladaptive responses due to the chronification of migraine attacks.
\end{abstract}

Keywords: Chronic migraine, Episodic migraine, Nociceptive blink reflex, Pain-related evoked potential, Trigeminal pathway

\section{Background}

Chronic migraine $(\mathrm{CM})$ is a disabling neurological condition, accepted by the main body of the current beta version of the International Classification of Headache Disorders-3 (ICHD-3 $\beta$ ) [1]. This classification indicates that the chronicity of the disorder is not a complication of migraine, but rather a transformation from an episodic to a chronic disorder. Approximately $3 \%$ of individuals with episodic migraine (EM) progress to $\mathrm{CM}$ over the course of a year [2]. Despite the spectrum

\footnotetext{
*Correspondence: deepfoci@hallym.or.kr

Department of Neurology, Chuncheon Sacred Heart Hospital, Hallym

University College of Medicine, 153 Gyo-dong, Chuncheon-si, Gangwon-do 200-704, Republic of Korea
}

conceptualization of migraine, there are important distinctions between EM and CM. Several studies have provided data on differences in symptoms, comorbidity profiles, disabilities, and treatment responses of CM versus EM [3-6]. Moreover, differences in pathophysiology and functional correlates observed in electrophysiological and imaging studies have been noted [7]. Although the precise mechanisms underlying headache chronification from EM to CM are not fully understood, the central sensitization and dysfunctional pain control systems are thought to be involved [8].

The trigeminal nociceptive system plays a pivotal role in the pathophysiology of migraine. The use of neurophysiological methods to explore pain-related circuits is 
an important aid in headache research. To increase nociceptive sensitivity, previous studies have developed a novel technique of selective electrical transcutaneous nociceptive fiber stimulation using a custom concentric planar electrode. The concentric electrode allows quantitative measurements of trigeminal nociception transmission and is highly sensitive to changes in trigeminal nociception [9-13]. Investigations of trigeminal nociceptive pathways during ictal phases have been recorded in $\mathrm{EM}$, and these data suggest temporary and specific sensitization of central trigeminal neurons during acute migraine attacks $[9,14]$. In addition, the possibility that increased excitability of the trigeminal pathways may persist during the interictal period has been proposed in EM [15]; however, investigations of trigeminal sensory pathways in $\mathrm{CM}$, particularly in regard to nociceptive processing, are rare. One previous study did not observe any changes [16], whereas other studies observed abnormal excitability $[13,17]$. These inconsistencies may result from the use of different study techniques, such as the conventional blink reflex, or different study groups, such as in individuals with combined medicationoveruse headaches. It has been assumed that these abnormalities would be more marked in $\mathrm{CM}$, and that there are distinctions between EM and CM.

Thus, we hypothesized that the nociceptive trigeminal pathway may be altered to varying degrees in patients with EM and CM. We assessed trigeminal nociceptive processing in patients with EM and $\mathrm{CM}$ during the interictal period compared to healthy controls to investigate the presence or absence of facilitation processes in the patient group. To determine whether there were differences in trigeminal pain processing at the brainstem and cortical levels, we used the nociceptive blink reflex (nBR) and pain-related evoked potentials (PREP) in EM and CM patients.

\section{Methods}

Subjects

This study collected data from patients with EM and CM treated in the headache clinic of a university hospital between October 2014 and June 2015. All participants were between 20 and 60 years of age, and only females were included to eliminate age and gender bias. They were examined and classified according to the ICHD- $3 \beta$ by a board-certified neurologist based on patient history, a neurologic examination, and neuroimaging study. Additionally, patients were required to have at least a 1-year migraine history prior to enrollment, to exclude other primary headaches. 30 patients with CM and 38 patients with EM were investigated in a casecontrol design. The control group consisted of 40 agematched female volunteers. We recruited the control group via advertisements such as posted notices in the hospital. Control participants had to be devoid of headaches for at least 3 months prior to enrollment, have no personal or family history of migraine, and have no more than an occasional mild headache ( $<5$ times per year); we also included only those participants who had not sought medical treatment for headaches. Exclusion criteria included subjects with prophylactic daily medication to prevent headaches, concomitant medicationoveruse headache based on ICHD-3 $\beta$, neurological disorders, any other systemic disease, history of Bell's palsy, somatic or psychiatric illness (e.g., depression, anxiety disorder), use of oral contraceptives, and pregnancy.

The patients with migraine were asked to complete a headache questionnaire during the last one month. Headache frequency (days/month) by the number of days with headaches for a month, duration (hours/day) by the sum of the total hours of headaches per attack, and intensity (numeric rating scale [NRS]: $0=$ no pain to $10=$ unbearable pain) by calculating the mean of the NRS for the days with headaches were described. All participants were instructed to refrain from caffeine, nicotine, and alcohol for at least $24 \mathrm{~h}$ prior to testing. This study protocol and informed consent form were reviewed and approved by the local Institutional Review Board (Institutional Review Board/Ethics Committee of Hallym University Chuncheon Sacred Heart Hospital). Written informed consent was obtained from all participants prior to enrollment in the study.

\section{Experimental procedure}

The recording sessions were conducted at the same time of day (2-5 PM) by expert neurophysiologists $(\mathrm{J}-\mathrm{H}, \mathrm{J})$ blind to the clinical diagnosis of the subjects. Recordings were performed using a Nicolet EDX EMP/EP machine (Natus Neurology, Middleton, WI, USA). Patients with migraine underwent testing during headache-free days. Specifically, recordings in EM patients were obtained interictally at least 2 days after the last and before the next migraine attack. All CM patients underwent the recordings in the interictal period (at least 2 days before and after a typical migraine attack), but a current background mild headache $(\mathrm{NRS}<3)$ was allowed. Following the test, patients were contacted by telephone and excluded from the study if they had experienced a migraine attack within 2 days of the recording. The participants were in a lying position with their eyes closed during the recordings. Two planar concentric surface stimulating electrodes were used for nociceptive stimulation (inomed Medizintechnik GmbH, Emmendingen, Germany, http://www.inomed.com).

Individual sensory (Is) and pain (Ip) perception thresholds were defined as the minimum stimulation intensity perceived as tactile and painful, respectively. We first determined sensory and pain threshold on both sides of 
the forehead using ascending and descending single pulses in $0.1 \mathrm{~mA}$ steps, with a duration of $0.5 \mathrm{~ms}$ and an interstimulus interval of 15-20 s (randomized interval). For patients' comfort and to avoid a lengthy procedure, we performed unilateral stimulation for the nBR and PREP recordings. The concentric electrode was placed on the left lower forehead, approximately $10 \mathrm{~mm}$ above the supraorbital foramen, to stimulate the supraorbital nerve. $\mathrm{nBR}$ and PREP were recorded simultaneously following trigeminal stimulation of the forehead A fixed stimulation intensity (SI) of $1.5 \times$ Ip was used to evoke the $\mathrm{nBR}$ and PREP. Trains of electrical stimulation composed of three pulses (each $0.5 \mathrm{~ms}$ in duration) with a $5 \mathrm{~ms}$ interpulse interval were applied to further increase nociceptive specificity [12], and stimulation intensities > $2 \mathrm{~mA}$ were excluded to minimize the risk of $\mathrm{A} \beta$ fiber co-activation [10]. Repeated stimulation of the train pulse was delivered at random intervals of $18-22 \mathrm{~s}$ to obtain at least 11 consecutive responses. Surface recording electrodes for $\mathrm{nBR}$ were placed on the infraorbital area (active) and at the base of the nose with a $2 \mathrm{~Hz}$ to $1 \mathrm{kHz}$ band-pass filter (sampling rate $2.5 \mathrm{kHz}, 150 \mathrm{~ms}$ sweep length, $200 \mathrm{~ms}$ analysis time, and $100 \mu \mathrm{V}$ sensitivity), which was used for recording. PREP was recorded with electrodes placed at $\mathrm{Cz}$ linked to both earlobes (A1-A2) based on the international 10-20 system.

\section{Analysis}

An investigator who was blind to the diagnosis performed signal analyses. All recordings were averaged offline using the Synergy Reader software, version 20.1 (Natus Neurology). The first recording was excluded from signal analysis to avoid contamination with startle response.

For the nBR recording, the 10 responses were rectified and averaged offline. The onset latency was visually determined as the initial point from the baseline (ms), and the AUC was determined between $27 \mathrm{~ms}$ and $87 \mathrm{~ms}$ of the R2 component ( $\left.\mathrm{mV}^{*} \mathrm{~ms}\right)$ [18]. The following parameters were measured: the onset latency (ms), the mean amplitude of the root mean square (RMS, $\mu \mathrm{V})$, and the area under the curve (AUC, $\mathrm{mV}^{*} \mathrm{~ms}$ ) values from the ipsilateral and contralateral R2 (iR2 and cR2).
For the PREP recording, the 10 responses were averaged offline. The negative (N1) and positive (P1) peak latencies and peak-to-peak amplitudes (PPA) were analyzed. The following parameters were measured: the peak latency of N1 and P1 (ms) and the mean of the PPA $(\mu \mathrm{V})$.

\section{Statistics}

Statistical analyses were performed using SPSS software ver. 21.0 for Windows (SPSS, Inc., Chicago, IL, USA). All values are reported as means and standard deviations. The Kruskal-Wallis test was used to compare the thresholds and parameters of nBR and PREP between groups. The Mann-Whitney $U$ test for multiple comparisons was performed for the post-hoc analyses. Spearman's correlation analysis was used to explore the relationship between electrophysiologic parameters and headache parameters (i.e., frequency, duration, and intensity). Results were considered significant at a $p$-value $<0.05$.

\section{Results \\ Clinical characteristics}

No significant differences in age were noted (EM: 40.95 years, CM: 43.07 years, controls: 43.73 years). The frequency of headaches and the duration of the headaches according to the headache questionnaire were 4.81 days/month and $14.71 \mathrm{~h} /$ day, in patients with EM and 20.03 days/month and $16.00 \mathrm{~h} /$ day, respectively, in patients with $\mathrm{CM}$. The mean intensity per episode (NRS) was 7.74 in patients with EM and 7.96 in patients with CM. The duration of having the headache condition was 9.93 years and 14.13 in patients with EM and $\mathrm{CM}$, respectively (Table 1 ).

\section{Sensory and pain thresholds}

The mean values for sensory and pain perception thresholds $(\mathrm{mA})$ were as follows: $0.20 \pm 0.09,0.78 \pm 0.30$ in EM, $0.19 \pm 0.05,0.77 \pm 0.26$ in $\mathrm{CM}$, and $0.20 \pm 0.10$, $0.84 \pm 0.35$ in controls. Patients with CM displayed lower sensory and pain perception thresholds during the recording; however, no significant differences were noted

Table 1 Demographic data and headache features

\begin{tabular}{lllll}
\hline & EM & CM & Control \\
& $n=38$ & $n=30$ & $n=40$ \\
\hline Age (years) & $40.95 \pm 10.00$ & $43.07 \pm 11.09$ & $43.73 \pm 11.75$ & NS \\
Frequency (days/month) & $4.81 \pm 3.30$ & $20.03 \pm 4.39$ & - & 0.00 \\
Duration (hours/day) & $14.71 \pm 8.44$ & $16.00 \pm 8.46$ & - & NS \\
Intensity (NRS: 0-10) & $7.74 \pm 1.91$ & $7.96 \pm 1.67$ & - & NS \\
Disease duration (years) & $9.93 \pm 9.06$ & $14.13 \pm 10.00$ & - & 0.07 \\
\hline
\end{tabular}

Values are expressed as mean \pm standard deviation

$C M$ chronic migraine, EM episodic migraine, NS not significant, NRS numeric rating scale 
between subject groups. The mean values for pain stimulation intensity of each group was 1.17 (confidence interval, $\mathrm{CI}, 0.32-1.98)$ in $\mathrm{EM}, 1.16$ (CI, 0.59-1.95) in $\mathrm{CM}$, and 1.27 (CI, 0.32-1.98) in controls.

\section{Nociceptive blink reflex}

The $\mathrm{nBR}$ results related to trigeminal nociceptive processing at the brainstem level are summarized in Table 2 . Examples of an $\mathrm{nBR}$ recording from a patient with EM, a patient with $\mathrm{CM}$, and a healthy control are shown in Fig. 1. The mean amplitude (RMS) and AUC values from the ipsilateral and contralateral R2 components (iR2 and cR2) were significantly lower in CM patients than in control and EM patients. Additionally, significantly prolonged latencies of the $\mathrm{R} 2$ component were seen in patients with $\mathrm{CM}$ ( $\mathrm{iR} 2$ in $\mathrm{CM}$ vs. controls, $\mathrm{iR} 2$ and cR2 in CM vs. EM, $p<0.05)$. However, EM patients displayed significantly decreased latencies and larger amplitudes and AUC values of both R2 components in $\mathrm{nBR}(p<0.05)$.

\section{Pain-related evoked potentials}

The PREP results regarding trigeminal nociceptive processing at the cortical level are summarized in Table 3. Examples of a PREP recording from patients with EM and $\mathrm{CM}$ and a healthy control are shown in Fig. 1. EM patients displayed significantly decreased latencies (both $\mathrm{N} 1$ and P1), with larger right amplitude (PPA), compared with controls $(p<0.05)$. Additionally, CM patients showed significantly decreased latencies (left N1, left and right P1), with larger right amplitude (PPA), compared with controls $(p<0.05)$. However, no significant differences were found in the PREP parameters between patients with EM and CM.

\section{Correlations between headache parameters and electrophysiologic parameters}

To explore possible clinical-electrophysiological correlations, we examined the correlations between headache parameters, including frequency, duration, and intensity, and $\mathrm{nBR}$ and PREP parameters. Although the latency of the $\mathrm{R} 2$ component in the $\mathrm{nBR}$ was positively correlated with the frequency of headache, the amplitude and AUC from the $\mathrm{R} 2$ component in the $\mathrm{nBR}$ were negatively correlated with the frequency of headache parameters in the migraine group $(p<0.01)$ (Table 4$)$. However, apart from these, no significant correlations were noted between $\mathrm{nBR}$ and PREP parameters and other headache parameters.

\section{Discussion}

Our results showed R2 suppression at the brainstem level (nBR) and facilitation at the cortical level (PREP) of trigeminal pain processing in CM patients, and facilitation at both the brainstem and cortical levels in EM patients. In addition, for the $\mathrm{nBR}$, a significant correlation between headache frequency and R2 suppression (prolonged latency, low amplitude, low AUC) was demonstrated in migraineurs.

A variety of nociceptive stimuli are able to elicit PREP at the cortical level and can be used to assess spinothalamocortical pain-temperature pathways $[19,20]$. Previous clinical studies have demonstrated the facilitation of the trigeminal nociceptive system in a pain-free state with event-related potentials following $\mathrm{CO}_{2}$ laser stimulation (LEP) in CM and chronic tension-type headache [21, 22]. In patients with medication overuse headache, activation of trigeminal and somatic PREP, but not of nBR was also found [13]. However, these central facilitation changes at the cortical level have not been observed in other chronic headache disorders, such as hypnic headache [23]. Thus, it is not clear whether this observed phenomenon is the cause or the consequence of the headache chronification.

In our study, facilitation of trigeminal PREP during the inter-ictal period was observed in both the EM and CM groups. The cingulate cortex has been thought to play a major role in cortical plasticity as the main generator of PREP or LEP [24, 25]. Patients with EM have shown deficient habituation with LEP represented by an abnormal excitability of cortical areas during interictal phase [26]. Several studies have shown modulation of the habituation deficit in LEP induced by preventive medication and by highfrequency repetitive transcranial magnetic stimulation $[27,28]$. This deficient habituation in the LEP was also

Table 2 Nociceptive blink reflex parameters

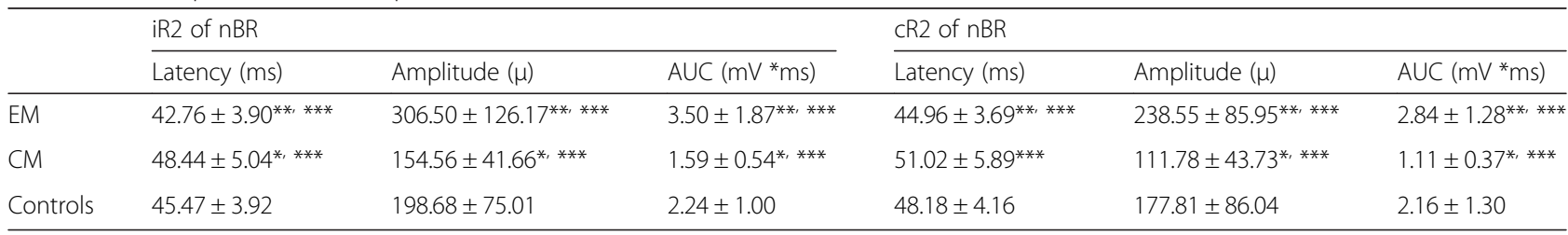

${ }^{*} p<0.05$ between $\mathrm{CM}$ and controls; ** $p<0.05$ between EM and controls; ${ }^{* * *} p<0.05$ between CM and EM. $P$-values were determined using the Mann-Whitney $U$ test

All values are expressed as means and standard deviations. $E M$ episodic migraine, $C M$ chronic migraine, $n B R$ nociceptive blink reflex, iR2 ipsilateral R2 component, CR2 contralateral R2 component, $A U C$ area under the curve 

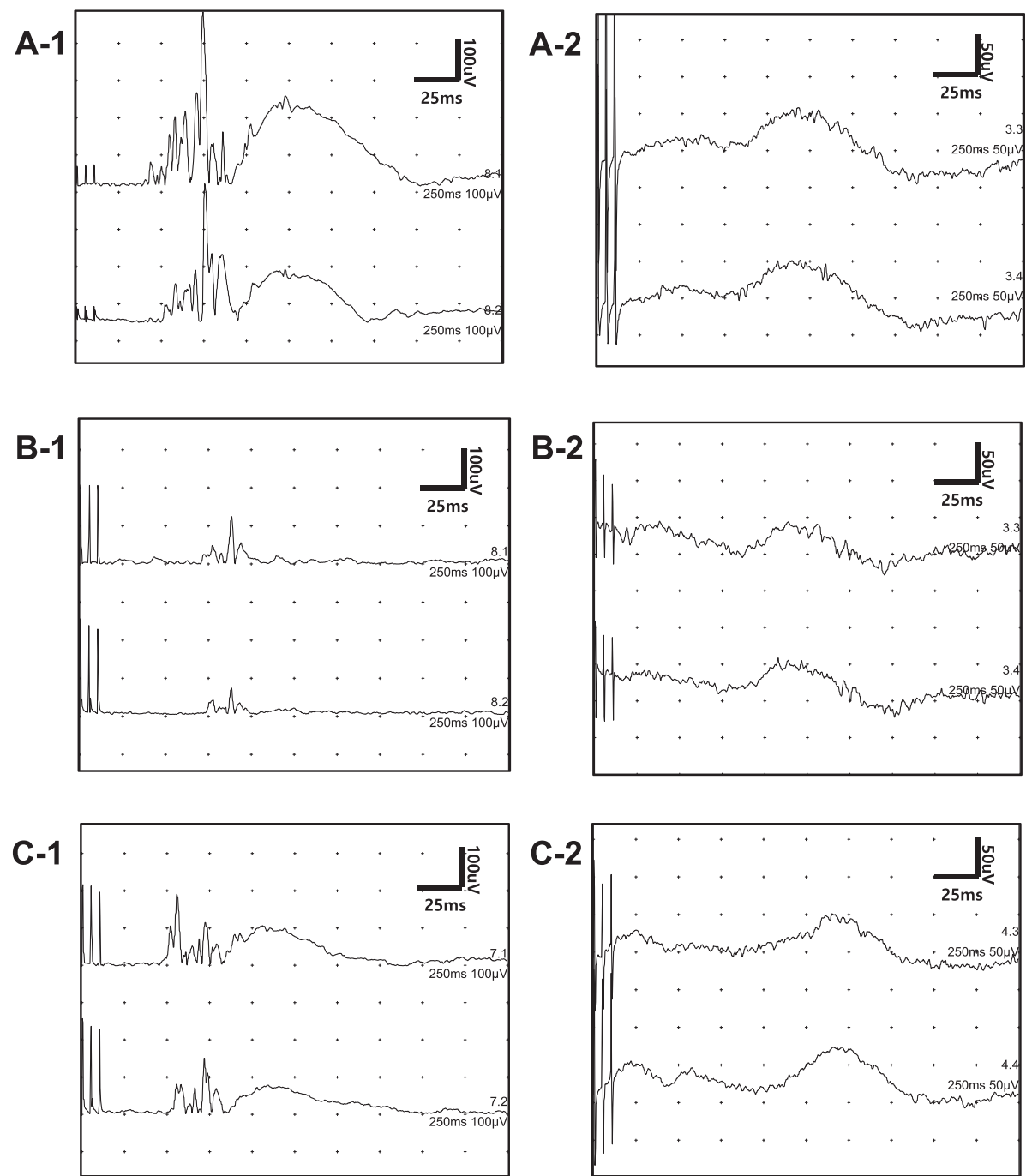

Fig. 1 Examples of the $n B R$ and PREP in patients with episodic and chronic migraine and controls. A-1 nBR in an EM patient, $\mathbf{A}-\mathbf{2}$ PREP in an EM patient, B-1 nBR in a CM patient, B-2 PREP in a CM patient, C-1 nBR in a control patient, C-2 PREP in a control patient. nBR, nociceptive blink reflex; PREP, pain-related evoked potential; EM, episodic migraine; $C M$, chronic migraine

partly restored after treatment of medication overuse headache [29]. These experimental studies reflect a modification in pain-processing pathways, which may constitute an important dysfunction, that probably forms the background in migraineurs. Thus, the facilitation of trigeminal nociception at the cortical level may be specific to migraineurs rather than to the chronification of pain.

The R2 component of the blink reflex reflects the excitability of brainstem interneurons and the function of synaptic transmission in the brainstem [30]. Previous studies have reported that the R2 component of the nBR

Table 3 Pain-related evoked potential parameters

\begin{tabular}{|c|c|c|c|c|c|c|}
\hline \multirow{3}{*}{. } & \multicolumn{3}{|l|}{ Left PREP } & \multicolumn{3}{|l|}{ Right PREP } \\
\hline & N1 latency (ms) & P1 latency (ms) & PPA $(\mu)$ & N1 latency (ms) & P1 latency (ms) & PPA $(\mu)$ \\
\hline & $122.62 \pm 9.71^{* *}$ & $169.82 \pm 9.70^{* *}$ & $63.76 \pm 13.22$ & $121.37 \pm 10.09^{* *}$ & $169.56 \pm 10.61^{* *}$ & $63.75 \pm 13.35^{* *}$ \\
\hline $\mathrm{CM}$ & $125.25 \pm 8.71^{*}$ & $172.83 \pm 10.43^{*}$ & $61.05 \pm 15.65$ & $125.95 \pm 8.46$ & $172.47 \pm 10.43^{*}$ & $59.13 \pm 15.74^{*}$ \\
\hline Controls & $130.71 \pm 6.84$ & $179.10 \pm 8.50$ & $59.48 \pm 14.25$ & $130.14 \pm 6.94$ & $178.59 \pm 8.05^{* *}$ & $54.63 \pm 13.91$ \\
\hline
\end{tabular}

${ }^{*} p<0.05$ between CM and controls; ${ }^{* *} p<0.05$ between EM and controls; there are no statistical differences between CM and EM for PREP parameters. $P$-values were determined using the Mann-Whitney $U$ test. All values are expressed as means and standard deviations

EM episodic migraine, CM chronic migraine, PREP pain-related evoked potential, PPA peak-to-peak amplitude, AUC area under the curve 
Table 4 Correlation coefficients between headache characteristics and parameters of the nBR and the PREP in migraineurs

\begin{tabular}{|c|c|c|c|}
\hline & \multicolumn{3}{|c|}{ Correlation coefficient $(r)$ for the nBR parameters } \\
\hline & Latency & Amplitude & AUC \\
\hline \multirow[t]{2}{*}{ Frequency of headache attacks (days) } & $0.497(\mathrm{iR} 2)^{* *}$ & $-0.588(\mathrm{iR} 2)^{* *}$ & $-0.521(\mathrm{iR} 2)^{* *}$ \\
\hline & $0.469(c R 2)^{* *}$ & $-0.634(c R 2)^{* *}$ & $-0.626(c R 2)^{* *}$ \\
\hline Duration of headache attacks (hours) & NS & NS & NS \\
\hline Pain intensity of headache attacks (NRS) & NS & NS & NS \\
\hline
\end{tabular}

Spearman's correlation test $(r),{ }^{* *} p<0.01$. EM episodic migraine, $C M$ chronic migraine, PREP pain-related evoked potential, NRS numeric rating scale, NS non-significant, $n B R$ nociceptive blink reflex, iR2 ipsilateral R2 component, $C R 2$ contralateral R2 component, AUC area under the curve, PREP pain-related evoked potential, PPA peak-to-peak amplitude

by nociceptive stimuli was more than six times greater during migraine attacks [9]; these facilitation changes are specific to episodic migraines and act as the central driving force for migraine attacks [14]. Several studies using neurophysiologic tools to explore the trigeminal system during the interictal period found that patients with EM have a lack of habituation of nBR and enhanced $\mathrm{R} 2$ recovery of $\mathrm{BR}$ and corneal reflex abnormality $[15,31$, 32]. Our results showing $R 2$ facilitation of $n B R$ between attacks in EM patients are consistent with these findings. These phenomena suggest that sensitization of the trigeminal system may persist interictally. Another interesting finding is that asymptomatic individuals with a family history of migraine present the same nBR abnormalities as patients with full-brown migraine between attacks [33]. On the other hand, another study found normal the R2 recovery curves between attacks in EM patients and these results do not support persistent interictal sensitization in the spinal trigeminal sensory system [34].

However, in terms of the nBR, R2 suppression occurred in the CM group, which differed from the EM group in our study in this regard. Additionally, each parameter of the nBR (latency, amplitude, and AUC) was positively or negatively correlated with headache frequency in migraineurs. Thus, the degree of R2 suppression seems to be correlated with the number of headache days. Brainstem interneuronal excitability is assumed to be under the control of rostral structures, and the R2 component of the blink reflex is largely influenced by suprasegmental control, that comes mainly from the cerebral cortex and basal ganglia [35, 36]. The descending pain modulation system might be responsible for keeping the primary value of the $\mathrm{nBR}$ within certain physiological boundaries with respect to homeostatic neuronal plasticity [37]. These networks of cortical and subcortical structures with modulatory nociceptive and antinociceptive functions become abnormally activated during, or even between, migraine attacks [38]. During repeated migraine attacks, modification of this network can occur. Therefore, excessive R2 suppression at the brainstem level may be related to an impaired or dysfunctional descending pain modulation system. These findings may relate to adaptive or dysregulated/maladaptive mechanisms within the context of the allostatic load model [39].

Excitability changes in the blink reflex circuit can also occur as a consequence of damage in the neural structures along the blink reflex circuit [36]. Several neuroimaging studies on migraineurs reported morphological and functional abnormalities in the brainstem [40-43]. In particular, EM patients demonstrated activation of the rostral brainstem during the interictal phase in functional MRI and exhibited increased density in the periaqueductal gray matter in structural imaging using voxel-based morphometry $[42,43]$. MRI findings of iron accumulation in the brainstem have been correlated with both duration of illness and frequency of attacks in migraineurs [44]. Additionally, CM patients showed persistent brainstem dysfunction in positron emission tomography evaluations and brainstem atrophy in highresolution anatomical MRI images $[45,46]$. A functional imaging study using ${ }^{1} \mathrm{H}$-magnetic resonance spectroscopy showed an increased $\mathrm{N}$-acetyl aspartate/creatinine (NAA/Cr) ratio suggestive of neuronal hypertrophy at the dorsal pons in EM compared with controls. Headache frequency and intensity were negatively correlated with the NAA/Cr ratio. These findings suggest that after an initial response of hypertrophy in EM, there may be neuronal loss in CM in these regions [47]. Thus, these plastic structural changes according to migraine status may influence the R2 facilitation or suppression of the $\mathrm{nBR}$ in migraineurs. Repeated episodes of central sensitization may be associated with neuronal damage at the brainstem level, with resultant poor modulation of pain, and migraine progression [48]. However, it is currently unclear whether these structural brain changes seen in migraineurs are the cause or the result of headaches.

Our study had several limitations. Patients at a specialized headache clinic were recruited, and the size of the sample was small. Additionally, the study used a crosssectional design that provided limited information. To further understand the differences between EM and CM, longitudinal electrophysiological follow-up studies are warranted. Moreover, we used only unilateral stimulation for recording to avoid an excessively lengthy 
procedure and to enhance patients' comfort. We believe that these decisions are probably not relevant to the interpretation of our results, because none of our patients had fixed unilateral migraine attacks, and there were no side differences in stimulus detection and pain threshold measurements. In addition, only females were included in the present study because of potential effects of sex on the blink reflex [49]; therefore, we are unable to generalize to men. A larger study sample will be required for a better and improved understanding of the relationship between measured electrophysiological factors and clinical findings.

\section{Conclusions}

In conclusion, this study provides electrophysiological evidence that excitability of nociceptive-specific trigeminal pathways is different between EM and CM. Facilitation of trigeminal nociceptive processing was observed in EM, whereas R2 suppression at the brainstem level and additional central facilitation changes at the cortical level were seen in CM. Facilitation along trigeminal nociceptive processing in EM patients was associated with migraine-specific hyperexcitability due to intrinsically increased excitability or impaired intra-inhibitory mechanisms. In CM, additional R2 suppression at the brainstem level may be related to impaired or dysfunctional descending pain modulation. These changes in $\mathrm{CM}$ are suggestive of adaptive or maladaptive responses due to repetitive episodes of migraine attacks.

\begin{abstract}
Abbreviations
AUC: area under the curve; CM: chronic migraine; CR2: contralateral R2 component; EM: episodic migraine; ICHD-3 $\beta$ : beta version of the International Classification of Headache Disorders-3; Ip: pain perception threshold; iR2: ipsilateral R2 component; Is: sensory perception threshold; N1: negative peak; nBR: nociceptive blink reflex; NRS: numeric rating scale; P1: positive peak; PPA: peak-to-peak amplitude; PREP: pain-related evoked potentials; RMS: root mean square; SI: stimulation intensity.
\end{abstract}

\section{Competing interests}

The authors declare that they have no competing interests.

\section{Authors' contributions}

Conception and design of the experiments: JHS. Performance of experiments: CHK, HCC, and JHS. Data analysis: JHS. Writing of the manuscript: JHS. All authors read and approved the final manuscript.

Received: 12 February 2016 Accepted: 12 April 2016

Published online: 16 April 2016

\section{References}

1. Headache Classification Committee of the International Headache Society (2013) The international classification of headache disorders, 3rd edition (beta version). Cephalalgia 33:629-808

2. Bigal ME, Lipton RB (2008) Concepts and mechanisms of migraine chronification. Headache 48(1):7-15

3. Blumenfeld AM, Varon SF, Wilcox TK, Buse DC, Kawata AK, Manack A, Goadsby PJ, Lipton RB (2011) Disability, HRQoL and resource use among chronic and episodic migraineurs: results from the International Burden of Migraine Study (IBMS). Cephalalgia 31(3):301-315
4. Buse D, Manack A, Serrano D, Reed M, Varon S, Turkel C, Lipton R (2012) Headache impact of chronic and episodic migraine: results from the American Migraine Prevalence and Prevention study. Headache 52(1):3-17

5. Ferrari A, Leone S, Vergoni AV, Bertolini A, Sances G, Coccia CP, Ottani A, Pinetti D, Sternieri E (2007) Similarities and differences between chronic migraine and episodic migraine. Headache 47(1):65-72

6. Katsarava Z, Manack A, Yoon MS, Obermann M, Becker H, Dommes P, Turkel C, Lipton RB, Diener HC (2011) Chronic migraine: classification and comparisons. Cephalalgia 31(5):520-529

7. Aurora SK, Kulthia A, Barrodale PM (2011) Mechanism of chronic migraine. Curr Pain Headache Rep 15(1):57-63

8. Coppola G, Schoenen J (2012) Cortical excitability in chronic migraine. Curr Pain Headache Rep 16(1):93-100

9. Kaube H, Katsarava Z, Przywara S, Drepper J, Ellrich J, Diener HC (2002) Acute migraine headache: possible sensitization of neurons in the spinal trigeminal nucleus? Neurology 58(8):1234-1238

10. Kaube H, Katsarava Z, Kaufer T, Diener H, Ellrich J (2000) A new method to increase nociception specificity of the human blink reflex. Clin Neurophysiol 111(3):413-416

11. Katsarava Z, Ellrich J, Diener HC, Kaube H (2002) Optimized stimulation and recording parameters of human 'nociception specific' blink reflex recordings. Clin Neurophysiol 113(12):1932-1936

12. Giffin NJ, Katsarava Z, Pfundstein A, Ellrich J, Kaube H (2004) The effect of multiple stimuli on the modulation of the 'nociceptive' blink reflex. Pain 108(1-2):124-128

13. Ayzenberg I, Obermann M, Nyhuis P, Gastpar M, Limmroth V, Diener HC, Kaube H, Katsarava Z (2006) Central sensitization of the trigeminal and somatic nociceptive systems in medication overuse headache mainly involves cerebral supraspinal structures. Cephalalgia 26(9):1106-1114

14. Katsarava Z, Lehnerdt G, Duda B, Ellrich J, Diener HC, Kaube H (2002) Sensitization of trigeminal nociception specific for migraine but not pain of sinusitis. Neurology 59(9):1450-1453

15. Sandrini G, Proietti Cecchini A, Milanov I, Tassorelli C, Buzzi MG, Nappi G (2002) Electrophysiological evidence for trigeminal neuron sensitization in patients with migraine. Neurosci Lett 317(3):135-138

16. Brooks JB, Fragoso YD (2013) The blink reflex test does not show abnormalities in a large group of patients with chronic migraine. Arq Neuropsiquiatr 71(11):862-865

17. De Marinis M, Pujia A, Colaizzo E, Accornero N (2007) The blink reflex in 'chronic migraine. Clin Neurophysiol 118(2):457-463

18. Ellrich J, Treede RD (1998) Characterization of blink reflex interneurons by activation of diffuse noxious inhibitory controls in man. Brain Res 803(1-2): $161-168$

19. Arendt-Nielsen L, Andersen OK (2006) Electrophysiological assessment of pain. Suppl Clin Neurophysiol 59:241-249

20. Kakigi $R$, Inui $K$, Tamura $Y$ (2005) Electrophysiological studies on human pain perception. Clin Neurophysiol 116(4):743-763

21. de Tommaso M, Libro G, Guido M, Sciruicchio V, Losito L, Puca F (2003) Heat pain thresholds and cerebral event-related potentials following painful CO2 laser stimulation in chronic tension-type headache. Pain 104(1-2):111-119

22. de Tommaso M, Valeriani M, Guido M, Libro G, Specchio LM, Tonali P, Puca $F$ (2003) Abnormal brain processing of cutaneous pain in patients with chronic migraine. Pain 101(1-2):25-32

23. Holle D, Gaul C, Krebs S, Naegel S, Diener HC, Kaube H, Katsarava Z, Obermann M (2011) Nociceptive blink reflex and pain-related evoked potentials in hypnic headache. Cephalalgia 31(11):1181-1188

24. Bromm B (2004) The involvement of the posterior cingulate gyrus in phasic pain processing of humans. Neurosci Lett 361(1-3):245-249

25. Kakigi R, Watanabe S, Yamasaki H (2000) Pain-related somatosensory evoked potentials. J Clin Neurophysiol 17(3):295-308

26. Valeriani $M$, de Tommaso M, Restuccia D, Le Pera D, Guido M, lannetti GD, Libro G, Truini A, Di Trapani G, Puca F, Tonali P, Cruccu G (2003) Reduced habituation to experimental pain in migraine patients: a CO(2) laser evoked potential study. Pain 105(1-2):57-64

27. de Tommaso M, Brighina F, Fierro B, Francesco VD, Santostasi R, Sciruicchio V, Vecchio E, Serpino C, Lamberti P, Livrea P (2010) Effects of high-frequency repetitive transcranial magnetic stimulation of primary motor cortex on laser-evoked potentials in migraine. J Headache Pain 11(6):505-512

28. Di Clemente L, Puledda F, Biasiotta A, Vigano A, Vicenzini E, Truini A, Cruccu G, Di Piero V (2013) Topiramate modulates habituation in migraine: 
evidences from nociceptive responses elicited by laser evoked potentials. J Headache Pain 14:25

29. Ferraro D, Vollono C, Miliucci R, Virdis D, De Armas L, Pazzaglia C, Le Pera D, Tarantino S, Balestri M, Di Trapani G, Valeriani M (2012) Habituation to pain in "medication overuse headache": a CO2 laser-evoked potential study. Headache 52(5):792-807

30. Ellrich J (2000) Brain Stem Reflexes: Probing Human Trigeminal Nociception. News in Physiol Sci 15:94-97

31. Katsarava Z, Giffin N, Diener HC, Kaube H (2003) Abnormal habituation of 'nociceptive' blink reflex in migraine-evidence for increased excitability of trigeminal nociception. Cephalalgia 23(8):814-819

32. Shibata K, Yamane K, Iwata M (2006) Change of excitability in brainstem and cortical visual processing in migraine exhibiting allodynia. Headache 46(10):1535-1544

33. Di Clemente L, Coppola G, Magis D, Fumal A, De Pasqua V, Di Piero V, Schoenen J (2007) Interictal habituation deficit of the nociceptive blink reflex: an endophenotypic marker for presymptomatic migraine? Brain 130(Pt 3):765-770

34. Coppola G, Di Clemente L, Fumal A, Magis D, De Pasqua V, Pierelli F, Schoenen J (2007) Inhibition of the nociceptive R2 blink reflex after supraorbital or index finger stimulation is normal in migraine without aura between attacks. Cephalalgia 27(7):803-808

35. Esteban A (1999) A neurophysiological approach to brainstem reflexes. Blink reflex. Neurophysiol Clin 29(1):7-38

36. Valls-Sole J (2012) Assessment of excitability in brainstem circuits mediating the blink reflex and the startle reaction. Clin Neurophysiol 123(1):13-20

37. Bienenstock EL, Cooper LN, Munro PW (1982) Theory for the development of neuron selectivity: orientation specificity and binocular interaction in visual cortex. J Neurosci 2(1):32-48

38. Welch KM (2003) Contemporary concepts of migraine pathogenesis. Neurology 61 (8 Suppl 4):S2-S8

39. Borsook D, Maleki N, Becerra L, McEwen B (2012) Understanding migraine through the lens of maladaptive stress responses: a model disease of allostatic load. Neuron 73(2):219-234

40. Weiller C, May A, Limmroth V, Juptner M, Kaube H, Schayck RV, Coenen HH, Diener HC (1995) Brain stem activation in spontaneous human migraine attacks. Nat Med 1(7):658-660

41. Bahra A, Matharu MS, Buchel C, Frackowiak RS, Goadsby PJ (2001) Brainstem activation specific to migraine headache. Lancet 357(9261):1016-1017

42. Moulton EA, Burstein R, Tully S, Hargreaves R, Becerra L, Borsook D (2008) Interictal dysfunction of a brainstem descending modulatory center in migraine patients. PLoS ONE 3(11), e3799

43. Rocca MA, Ceccarelli A, Falini A, Colombo B, Tortorella P, Bernasconi L, Comi G, Scotti G, Filippi M (2006) Brain gray matter changes in migraine patients with T2-visible lesions: a 3-T MRI study. Stroke 37(7):1765-1770

44. Welch KM, Nagesh V, Aurora SK, Gelman N (2001) Periaqueductal gray matter dysfunction in migraine: cause or the burden of illness? Headache 41(7):629-637

45. Aurora SK, Barrodale PM, Tipton RL, Khodavirdi A (2007) Brainstem dysfunction in chronic migraine as evidenced by neurophysiological and positron emission tomography studies. Headache 47(7):996-1003, discussion 1004-1007

46. Bilgic B, Kocaman G, Arslan AB, Noyan H, Sherifov R, Alkan A, Asil T, Parman $Y$, Baykan B (2015) Volumetric differences suggest involvement of cerebellum and brainstem in chronic migraine. Cephalalgia. doi:10.1177/ 0333102415588328

47. Lai TH, Fuh JL, Lirng JF, Lin CP, Wang SJ (2012) Brainstem 1H-MR spectroscopy in episodic and chronic migraine. J Headache Pain 13(8):645-651

48. Bigal ME, Lipton RB (2006) When migraine progresses: transformed or chronic migraine. Expert Rev Neurother 6(3):297-306

49. Peddireddy A, Wang K, Svensson P, Arendt-Nielsen L (2006) Influence of age and gender on the jaw-stretch and blink reflexes. Exp Brain Res 171(4):530-540

\section{Submit your manuscript to a SpringerOpen ${ }^{\circ}$ journal and benefit from:}

- Convenient online submission

- Rigorous peer review

- Immediate publication on acceptance

- Open access: articles freely available online

- High visibility within the field

Retaining the copyright to your article

Submit your next manuscript at $\gg$ springeropen.com 